\title{
Effects of scleral buckling and encircling procedures on human optic nerve head and retinochoroidal circulation
}

\author{
Miyuki Nagahara, Yasuhiro Tamaki, Makoto Araie, Shuichiro Eguchi
}

\begin{abstract}
Aims-To study the effects of segmental scleral buckling and encircling procedures on tissue circulation in the human optic nerve head (ONH) and choroid and retina.

Methods-Using the laser speckle method, the normalised blur (NB) value, a quantitative index of tissue blood velocity, was measured every 0.125 seconds and averaged over three pulses in the optic nerve head $\left(\mathrm{NB}_{\mathrm{ONH}}\right)$ and choroid and retina $\left(\mathrm{NB}_{\text {ch-ret }}\right)$ in 10 patients with unilateral rhegmatogenous retinal detachment (mean age 52 (SD 17)). $\mathrm{NB}_{\mathrm{ONH}}, \mathrm{NB}_{\text {ch-ret }}$, and intraocular pressure (IOP) in both eyes, and blood pressure (BP) were measured before, and 1, 4, and 12 weeks after the scleral buckling and encircling procedure. Results- $-\mathrm{NB}_{\text {ch-ret }}$ on the buckled side was significantly reduced after surgery and smaller than that in the unoperated contralateral eye throughout the study period (ANOVA, $p<0.0001$ ). $\mathbf{N B}_{\text {ch-ret }}$ on the unbuckled side, in the foveal area, $\mathbf{N B}_{\mathrm{ONH}}$, IOP, and BP showed no significant change.

Conclusions-It was indicated that the segmental scleral buckling procedure with encircling elements decreased tissue blood velocity in the choroid and retina on the buckled side but caused no significant change on tissue circulation in other areas of the fundus or ONH.

(Br F Ophthalmol 2000;84:31-36)
\end{abstract}

Scleral buckling procedures are the treatment of choice for the majority of uncomplicated rhegmatogenous retinal detachments. Postoperative complications related to alterations in blood supply, such as anterior and posterior segment ischaemia which usually severely influence visual recovery, are rare but have been reported by several authors. ${ }^{1-4}$ It may be possible that subclinical ischaemia after retinal detachment surgery exists in many more cases than reported..$^{-11}$ Thus, the effects of scleral buckling procedures on ocular circulation, if they exist, have important clinical implications.

Studies in which the laser Doppler technique was used indicated that the pulsatile characteristics of the retinal arterial blood flow measured in the treated eyes were altered, ${ }^{5}$ and that arterial flow rates in the major temporal retinal arteries were, on average, 50\% lower in the treated eyes than in the unoperated contralateral eyes; removal of scleral buckling elements in two patients produced increases of $73 \%$ and $44 \%$ in arterial blood flow rates. ${ }^{6}$ One study using colour Doppler imaging indicated that scleral buckling procedures with encircling elements decreased average blood flow velocity in the central retinal artery by $35 \%$ on the first operative day and by $50 \% 1$ week postoperatively but did not affect that in the ophthalmic artery, ${ }^{7}$ whereas another study reported that the same surgery reduced the average blood flow velocity in the ophthalmic artery by $31 \% 2$ days after surgery. ${ }^{8}$ In patients with rhegmatogenous retinal detachment, the ocular pulse amplitude in the surgically treated eyes was reportedly lower by $53 \%$ compared with fellow eyes 6 months after scleral buckling. ${ }^{11}$ These findings suggest that scleral buckling decreases not only retinal but also choroidal and/or optic nerve head $(\mathrm{ONH})$ circulation because the ophthalmic artery is the origin of the arterial branches that supply blood to the eye. To date, however, there have been no reports on the effect of the scleral buckling procedure on tissue circulation in different parts of the human fundus - that is, in the $\mathrm{ONH}$, choroid in the foveal area, and choroid and retina on the buckled and unbuckled side.

We recently constructed an apparatus for non-contact, two dimensional estimation of ocular fundus tissue circulation, utilising the near infrared diode laser and the laser speckle phenomenon. The normalised blur (NB) value, a quantitative index of the tissue blood velocity, is measured non-invasively in a portion of the $\mathrm{ONH}$, choroid, and retina with reasonable reproducibility. ${ }^{12-15}$ Using this laser speckle method, we studied the effects of the scleral buckling procedure with encircling elements on tissue circulation in the $\mathrm{ONH}$, the choroid in the foveal area, and the choroid and retina on the buckled and unbuckled side in patients with rhegmatogenous retinal detachment before and after primary scleral buckling.

\section{Materials and methods}

SUBJECTS

From February 1996 to July 1996, 12 patients (mean age 52 years; range 21-7; four males and eight females) with unilateral, localised, quadrant rhegmatogenous retinal detachment repaired by uncomplicated scleral buckling and encircling procedures were consecutively enrolled. The entry criteria for the study were retinal detachment localised to the periphery of the fundus and not involving the macula, no other known retinal vascular disease, visual acuity correctable to at least 0.4 , adequate
Accepted for publication 11 August 1999 
pupillary dilatation, and clear media. Eyes which would need exoplants crossing the midline were not included in the study. The protocol of the study was approved by the ethics review committee of the University of Tokyo School of Medicine and written informed consent was obtained from each participant after full explanation of the procedure. No patient had any known haematological disorder. The primary surgeon (SE) was notified of the patient's enrolment into the study after the surgery.

Surgery was performed under local anaesthesia for all 12 patients by one of the authors (SE). Retinal breaks were identified in all patients and were treated with transscleral cryotherapy. A Mira No 240 silicone encircling element (Mira, Inc, Waltham, MA, USA), in conjunction with a silicone tyre exoplant, was used in all patients. The encircling element was secured to the sclera $13 \mathrm{~mm}$ posterior to the limbus with 5-0 Surgiron mattress sutures placed in the four quadrants between the muscles and fastened with a Mira No 270 sleeve with moderate tightness. The extent of the exoplant was from 1.5 to 2.5 quadrants and was consistent with the size and location of retinal breaks and co-existing lattice degeneration. Subretinal fluid was drained externally in every patient. Extraocular muscle disinsertion was not required for any patient. There were no intraoperative complications. At the end of the procedure, indirect ophthalmoscopy revealed retinal reattachment along with the absence of central retinal artery pulsations in all patients. In none of the patients was the eye believed to be excessively firm at the end of the operation. The height of the scleral buckle also was roughly judged at this point by the angle of bending the retinal vessel onto the segmental buckle by the primary surgeon (SE); about $30^{\circ}$ to $60^{\circ}$ designated as medium and $60^{\circ}$ to $90^{\circ}$ as high. (designated as low, medium, or high). The type and location of each exoplant was drawn in the chart after the procedure.

INSTRUMENT

A fundus camera was equipped with a diode laser (wavelength $808 \mathrm{~nm}$ ) and image sensor $(100 \times 100$ pixels $)$. The fundus where the laser beam was focused was observed by means of an infrared CCD camera. The scattered laser light was imaged on an image sensor corresponding to a field of $1.06 \times 1.06 \mathrm{~mm}$ (45 degree visual angle of the fundus camera) in the human fundus, on which a speckle pattern appeared. The difference between the average of the speckle intensity $\left(\mathrm{I}_{\text {mean }}\right)$ and the speckle intensity for successive scannings of the image speckles at the pixels on the sensor plane was calculated, and the ratio of $I_{\text {mean }}$ to this difference was defined as NB. ${ }^{12}{ }^{14} \mathrm{NB}$ is nearly equivalent to the reciprocal of the speckle contrast defined by Fercher and Briers. ${ }^{16}{ }^{17}$ The NB was calculated using a logic board every 0.125 seconds successively for a maximum of 7 seconds and divided into 50 colour coded levels, which were displayed as colour graphics on a colour monitor showing the two dimensional variation of the NB level over the field of interest. ${ }^{18}$ The average NB level $\left(\mathrm{NB}_{\mathrm{av}}\right)$ in any rectangular field of interest on a displayed colour map can be calculated and the change of $\mathrm{NB}_{\text {av }}$ over 7 seconds can be monitored at the same time. The maximum exposure of the retina with the present apparatus is below the permissible variable limits of American $\mathrm{Na}-$ tional Standard Institute (ANSI). ${ }^{18}$

NB MEASUREMENT IN HUMAN ONH AND CHOROID RETINA

The pupil was dilated with one drop of Mydrin M (0.4\% tropicamide, Santen Pharmaceutical, Osaka, Japan) 30 minutes before measurements were taken. During the measurement, the subject was asked to watch a target light; an electrocardiogram was monitored simultaneously. The image speckles from the measurement field located in the temporal site of the $\mathrm{ONH}$, corresponding to a field of $0.72 \times 0.72$ $\mathrm{mm}$ (30 degree visual angle of the fundus camera), were recorded. The average NB across the largest rectangular field in the measurement field free of visible surface vessels further averaged over three cardiac pulses when fixation was satisfactory, was calculated to obtain mean $\mathrm{NB}_{\mathrm{av}}$ from the ONH $\left(\mathrm{NB}_{\mathrm{ONH}}\right)$. The size of this rectangular field varied among subjects to avoid visible surface vessels, ranging from 0.15 $\times 0.22 \mathrm{~mm}(20 \times 30$ pixels $)$ to $0.37 \times 0.48 \mathrm{~mm}$ $(50 \times 65$ pixels $)$.

Image speckles from a field corresponding to a field of $1.06 \times 1.06 \mathrm{~mm}$ (45 degree visual angle of the fundus camera) were recorded and the average NB across the largest rectangular field free of visible surface retinal vessels was averaged as described above to obtain the mean $\mathrm{NB}_{\mathrm{av}}$ from the choroid and retina $\left(\mathrm{NB}_{\text {ch-ret }}\right)$. Although large choroidal vessels can be seen on the display, their contours were not as discrete as those of surface retinal vessels and the density of choroidal vessels are greater than surface visible retinal vessels. Therefore, we did not avoid visible choroidal vessels, while we did avoid visible retinal vessels. The size of this rectangular field varied among subjects to avoid surface visible retinal vessels, ranging from $0.48 \times 0.53 \mathrm{~mm}(45 \times 50$ pixels $)$ to $0.64 \times$ $0.80 \mathrm{~mm}(60 \times 75$ pixels $)$. Image speckles from the macula, corresponding to a field of $1.06 \times$ $1.06 \mathrm{~mm}$ (45 degree visual angle of the fundus camera), were recorded and the average NB across the foveal area free of retinal vessels was averaged as above to obtain the mean $\mathrm{NB}_{\text {av }}$ from the foveal area $\left(\mathrm{NB}_{\text {fovea }}\right)$. The size of this square field was $0.32 \times 0.32 \mathrm{~mm}(30 \times 30$ pixels).

The movement of the subject's eye in any direction during the measurement period was checked by the method previously described. ${ }^{14}$ It was also checked by inspecting the colour map and the time course plot of $\mathrm{NB}_{\mathrm{av}}$ taken every 0.125 seconds; when there was no eye movement during measurement, visible surface vessels did not change position on the colour map and the time course plot of $\mathrm{NB}_{\mathrm{av}}$ exhibited periodic fluctuations synchronised with cardiac pulse. ${ }^{18}$ On the other hand, when eye movement occurred, visible surface vessels in the colour map changed position according 


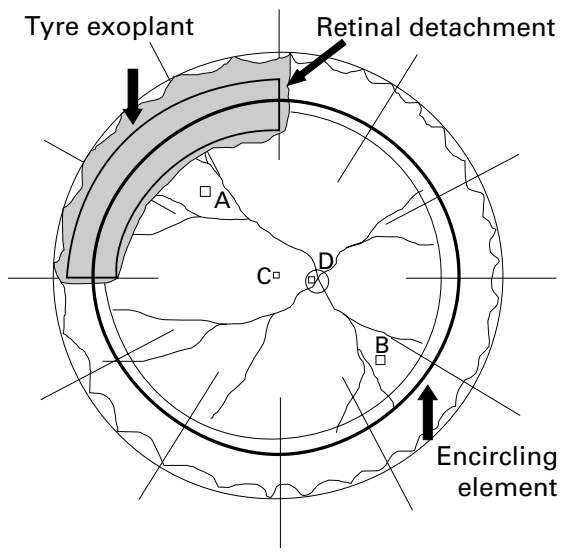

Figure 1 Measurement field of normalised blur (NB) in human choroid and retina and optic nerve head (ONH). Image speckles from a field on the buckled side $(A)$ and unbuckled side $(B)$ with no discrete retinal vessels visible in the operated eye and those on the mirror image sides of the unoperated contralateral eye were recorded to measure $N B$ in the choroid and retina. Image speckles from fovea and a field in temporal ONH, free of visible surface vessels, were recorded to measure $N B$ in the fovea $(C)$ and that in $O N H$ tissue (D).

to the eye movement. The coefficients of reproducibility for 5 minute and 24 hour interval measurements in normal humans were $11.7 \%$ and $13.0 \%$ for $\mathrm{NB}_{\mathrm{ONH}}$ and $8.7 \%$ and $9.7 \%$ for $\mathrm{NB}_{\text {ch-ret }}$, respectively. ${ }^{18}$

Image speckles from all subjects were digitally stored as colour maps. $\mathrm{NB}_{\mathrm{av}}$ was calculated by a blinded investigator (MN) for each subject in the same position and size of the rectangular field free of visible surface vessels by referring to a polaroid fundus picture and colour map.

\section{STUDY PROTOCOL}

The both eyes of a patient underwent identical assessment at each time point of measurement. The baseline measurements were carried out between 1100 and 1300 one day before surgery. After the pupil was dilated by one drop of Mydrin $\mathrm{M}$, the $\mathrm{NB}_{\mathrm{ONH}}$ and $\mathrm{NB}_{\text {fovea }}$ in both eyes were measured. The $\mathrm{NB}_{\text {ch-ret }}$ was measured in the eye with the retinal detachment in an area where the retina was not detached, outside the vascular arcade, and close to the area where the buckle was going to be located (the buckled side), and an area $180^{\circ}$ from the buckled side (unbuckled side) (Fig 1). Thereafter, the $\mathrm{NB}_{\text {ch-ret }}$ on the mirror image side of the unoperated contralateral eye in relation to the buckled or unbuckled side of the operated eye was measured. Polaroid fundus photographs were taken to record the sites of measurement. The IOP in both eyes and brachial arterial blood pressure were also measured. It took 5-10 minutes to complete all procedures in one eye.

During the prospective follow up period of 12 weeks, patients were examined 1,4 , and 12 weeks after the surgery and at each visit, brachial arterial blood pressure, and the IOP, $\mathrm{NB}_{\text {ONH }}, \mathrm{NB}_{\text {fovea }}$, and $\mathrm{NB}_{\text {ch-ret }}$ in both eyes were measured as described above. These NB measurements were performed in the same area as measured before surgery, referring to a fundus photograph and colour map of NB obtained before surgery to determine the preoperative locations. All measurements were performed between 1100 and 1300. The ocular perfusion pressure (OPP) was also calculated by:

$\mathrm{OPP}=2 / 3 \mathrm{BP}_{\mathrm{m}}-\mathrm{IOP}$ where $\mathrm{BP}_{\mathrm{m}}$ was mean brachial arterial blood pressure. All patients were monitored for evidence of anterior segment ischaemia or other postoperative complications.

\section{DATA ANALYSIS}

Statistical significance was determined with Student's $t$ test for paired variates. Analysis of variance (ANOVA) for the data obtained by sequential measurements was also used.

\section{Results}

Two of the 12 patients, who could not visit the hospital on the scheduled postoperative day, were excluded from the study and the following analysis was performed in 10 patients (Table 1). In both the operated and unoperated contralateral eyes, there was no significant change in IOP from the baseline and no bilateral differences at any of the time points (paired $t$ test, $\mathrm{p}>0.1$ ). There was no significant change from the baseline at any time point in mean blood pressure or OPP (paired $t$ test, $\mathrm{p}$ $>0.1$; Table 2).

There was no significant difference in the baseline $\mathrm{NB}_{\text {ch-ret }}, \mathrm{NB}_{\text {fovea }}$, and $\mathrm{NB}_{\mathrm{ONH}}$ at any of the corresponding sites between the operated and the unoperated contralateral eyes (Table 3). There was a significant approximately $40 \%$ decrease from the baseline in the $\mathrm{NB}_{\text {ch-ret }}$ on the buckled side of the operated eye (Table 4), while no significant change was observed in $\mathrm{NB}_{\text {ch-ret }}$ on the mirror image side of the unoperated contralateral eye. Further, no significant change was observed in the $\mathrm{NB}_{\mathrm{ONH}}$ and $\mathrm{NB}_{\text {fovea }}$, in both eyes, and $\mathrm{NB}_{\text {ch-ret }}$ on the unbuckled side of the operated eye and that on the mirror

Table 1 Patient characteristics

\begin{tabular}{|c|c|c|c|c|c|c|c|}
\hline \multirow[b]{2}{*}{ Patient } & \multirow[b]{2}{*}{ Age (years)/sex } & \multirow[b]{2}{*}{ Eye } & \multicolumn{4}{|c|}{ Visual acuity } & \multirow[b]{2}{*}{ Exoplant type } \\
\hline & & & Pre & 1 week & 4 week & 12 week & \\
\hline $1 \mathrm{AY}$ & $62 / \mathrm{M}$ & $\mathrm{R}$ & 0.8 & 0.5 & 1.0 & 0.8 & $240287(\mathrm{M})$ \\
\hline $2 \mathrm{SH}$ & $21 / M$ & $\mathrm{R}$ & 1.2 & 0.4 & 0.6 & 1.5 & $240287(\mathrm{H})$ \\
\hline $3 \mathrm{CY}$ & $75 / \mathrm{M}$ & $\mathrm{R}$ & 0.7 & 0.5 & 0.8 & 0.8 & $240287(\mathrm{M})$ \\
\hline $4 \mathrm{TS}$ & $68 / \mathrm{M}$ & $\mathrm{R}$ & 1.2 & 1.0 & 1.2 & 1.2 & $240287(\mathrm{H})$ \\
\hline $5 \mathrm{MO}$ & $46 / \mathrm{M}$ & $\mathrm{R}$ & 1.5 & 1.0 & 1.0 & 1.5 & $240220(\mathrm{H})$ \\
\hline $6 \mathrm{ST}$ & $53 / \mathrm{M}$ & $\mathrm{R}$ & 0.4 & 0.5 & 0.9 & 1.2 & $240220(\mathrm{H})$ \\
\hline $7 \mathrm{ME}$ & $23 / \mathrm{F}$ & $\mathrm{R}$ & 0.8 & 0.8 & 0.8 & 1.0 & $240287(\mathrm{H})$ \\
\hline $8 \mathrm{WY}$ & $63 / \mathrm{F}$ & $\mathrm{L}$ & 1.5 & 0.7 & 1.0 & 1.0 & $240219(\mathrm{M})$ \\
\hline $9 \mathrm{SK}$ & $41 / \mathrm{M}$ & $\mathrm{R}$ & 1.5 & 0.9 & 2.0 & 2.0 & $240220(\mathrm{H})$ \\
\hline $10 \mathrm{OT}$ & $49 / \mathrm{M}$ & $\mathrm{L}$ & 1.2 & 1.2 & 1.5 & 1.5 & $240287(\mathrm{H})$ \\
\hline
\end{tabular}

^Mira silicone element number (buckle height: $\mathrm{H}=$ high, $\mathrm{M}=$ medium). 
Table 2 Measurement of variables in patients with retinal detachment

\begin{tabular}{|c|c|c|c|c|}
\hline & Before & 1 week & 4 weeks & 12 weeks \\
\hline Intraocular pressure $(\mathrm{mm} \mathrm{Hg})$ (operated eye) & $13.4(4.7)$ & $13.8(3.8)$ & $13.8(3.8)$ & $13.1(3.5)$ \\
\hline Intraocular pressure (mm Hg) (unoperated contralateral eye) & $12.9(3.8)$ & $14.1(4.2)$ & $13.8(3.6)$ & $12.6(2.9)$ \\
\hline Mean blood pressure $(\mathrm{mm} \mathrm{Hg}) \dagger$ & $96(18)$ & $90(15)$ & $88(15)$ & $88(15)$ \\
\hline Ocular perfusion pressure $(\mathrm{mm} \mathrm{Hg}) \ddagger$ (operated eye) & $51(15)$ & $46(9)$ & $45(13)$ & $46(9)$ \\
\hline Ocular perfusion pressure $(\mathrm{mm} \mathrm{Hg}) \ddagger$ (unoperated contralateral eye) & $51(12)$ & $46(12)$ & $45(9)$ & $46(9)$ \\
\hline
\end{tabular}

Figures are mean (SD) in 10 patients. †Diastolic blood pressure $+1 / 3$ (systolic blood pressure—diastolic blood pressure), $\ddagger 2 / 3$ (mean blood pressure)—intraocular pressure There were no significant intergroup differences observed in any of the variables. There was no significant difference observed between the initial measurement and subsequent measurements in any of the variables.

Table 3 Baseline value in the $N B_{\text {ch-ret }}, N B_{\text {fovea }}$ and $N B_{O N H}$ in patients

\begin{tabular}{lll}
\hline & Operated eye & Unoperated contralateral eye \\
\hline $\mathrm{NB}_{\text {ch-ret }}$ (buckled side) & $25.1(8.8)$ & $23.9(6.5)$ \\
$\mathrm{NB}_{\text {ch-ret }}$ (unbuckled side) & $22.0(6.6)$ & $22.1(3.6)$ \\
$\mathrm{NB}_{\text {fovea }}$ & $28.2(5.8)$ & $27.5(6.5)$ \\
$\mathrm{NB}_{\mathrm{ONH}}$ & $23.6(4.4)$ & $25.2(7.6)$
\end{tabular}

Figures are mean (SD) in 10 patients. $\mathrm{NB}_{\text {ch-ret }}, \mathrm{NB}_{\text {fovea }}$ and $\mathrm{NB}_{\mathrm{ONH}}$ represent mean tissue blood velocity in the largest rectangular field in the measurement field free of visible surface vessels in choroid and retina, foveal area, and optic nerve head, respectively.

* Mirror image side of the buckled (unbuckled) side in the unoperated contralateral eye.

No significant bilateral difference is observed in any of variables (paired $t$ test, $\mathrm{p}>0.1$ ).

Table 4 Change in $N B_{\text {ch-ret, }} N B_{\text {fovea }}$ and $N B_{O N H}$

\begin{tabular}{lllll}
\hline & Before & 1 week & 4 weeks & 12 weeks \\
\hline Operated eye & & & & \\
$\Delta \mathrm{NB}_{\text {ch-ret }}$ (buckled side) & 0 & $-7.86(13.1)^{\star}$ & $-8.95(10.6)^{\star}$ & $-12.8(8.70)^{\star}$ \\
$\Delta \mathrm{NB}_{\text {ch-ret }}$ (unbuckled side) & 0 & $0.53(7.47)$ & $-1.66(5.81)$ & $-1.66(8.05)$ \\
$\Delta \mathrm{NB}_{\text {fovea }}$ & 0 & $-0.95(4.33)$ & $-2.73(5.74)$ & $-2.47(5.89)$ \\
$\Delta \mathrm{NB}_{\text {ONH }}$ & 0 & $-0.14(3.15)$ & $-0.10(3.24)$ & $-1.54(3.12)$ \\
Unoperated contralateral eye & 0 & $1.06(6.41)$ & $-0.03(5.88)$ & $1.87(4.75)$ \\
$\Delta \mathrm{NB}_{\text {ch-ret }}$ (buckled side) $\dagger$ & 0 & $0.19(3.66)$ & $-0.24(4.62)$ & $-0.40(1.72)$ \\
$\Delta \mathrm{NB}_{\text {ch-ret }}$ (unbuckled side) $\dagger$ & 0 & $0.93(4.21)$ & $-0.13(3.10)$ & $0.53(4.59)$ \\
$\Delta \mathrm{NB}_{\text {fovea }}$ & 0 & $-0.46(2.92)$ & $0.50(3.45)$ & $-0.71(3.15)$ \\
$\Delta \mathrm{NB}_{\text {ONH }}$ & 0 & & &
\end{tabular}

Figures are mean (SD) in 10 patients. $\mathrm{NB}_{\text {ch-ret }}, \mathrm{NB}_{\text {fovea }}$ and $\mathrm{NB}_{\mathrm{ONH}}$ represent mean tissue blood velocity in the largest rectangular field in the measurement field free of visible surface vessels in choroid and retina, foveal area, and optic nerve head, respectively. $\Delta \mathrm{NB}_{\text {ch-reet }}, \Delta \mathrm{NB}_{\text {fovea }}$ and $\Delta \mathrm{NB}_{\mathrm{ONH}}$ indicate difference in $\mathrm{NB}_{\text {ch-ret }}, \mathrm{NB}_{\text {fovea }}$ and $\mathrm{NB}_{\mathrm{ONH}}$ between before and each time point of measurement, respectively.

Bilateral difference is significant for $\Delta \mathrm{NB}_{\text {ch-ret }}$ in buckled side ( $\mathrm{p}<0.0001$, ANOVA), but not for in unbuckled side, $\Delta \mathrm{NB}_{\text {fovea }}$ and $\Delta \mathrm{NB}_{\mathrm{ONH}}$

* Significantly smaller than zero $(\mathrm{p}=0.0003 \sim 0.040)$

†Mirror image side of the buckled (unbuckled) side in the unoperated contralateral eye.

image side of the unoperated contralateral eye (Table 4). There were significant bilateral differences in the change in the $\mathrm{NB}_{\text {ch-ret }}$ from the baseline $\left(\Delta \mathrm{NB}_{\text {ch-ret }}\right)$ on the buckled side (ANOVA of repeated measurements, $\mathrm{p}<0.0001$ ), but not the unbuckled side (Table $4)$. The bilateral difference was not significant for the change in both $\mathrm{NB}_{\text {fovea }}$ and $\mathrm{NB}_{\mathrm{ONH}}$ from the baseline $\left(\Delta \mathrm{NB}_{\text {fovea }}\right.$ and $\Delta \mathrm{NB}_{\mathrm{ONH}}$, respectively; Table 4).

\section{Discussion}

METHOD

$\mathrm{NB}$ is primarily a quantitative index of tissue blood velocity. ${ }^{12}{ }^{14}$ In rabbits, when the ocular blood flow rate was artificially changed by increasing the $\mathrm{IOP}^{1214}$ or by intravenous injection of a calcium antagonist, ${ }^{15}$ averaged NB levels over the sampling field, $\mathrm{NB}_{\mathrm{av}}$, had a good correlation with the blood flow rate determined using the microsphere method in the retina or choroid. ${ }^{121415}$ These findings suggest that the NB also correlates with the blood flow rate in the choroid or retina, at least under certain conditions. According to Koelle et al, ${ }^{19}$ the penetration depth of near infrared laser (wavelength $811 \mathrm{~nm}$ ) in the cat optic nerve exceeds 1 $\mathrm{mm}$. When $\mathrm{ONH}$ blood flow rate in rabbits was artificially changed by a small amount of endothelin-1 or by inhalation of $10 \%$ carbon dioxide, $\mathrm{NB}_{\mathrm{av}}$ obtained from $\mathrm{ONH}$ tissue strongly correlated with the blood flow rate in the ONH determined using the hydrogen gas clearance method where a hydrogen electrode was inserted into the $\mathrm{ONH}$ tissue to a depth of approximately $0.7 \mathrm{~mm} .{ }^{13}$ Although blood flow rate measured by the hydrogen gas clearance method does not directly reflect the movement of red blood cells as the $\mathrm{NB}_{\text {av }}$ measured by the laser speckle method, these findings suggest that the NB correlates with the blood flow rate also in the $\mathrm{ONH}$, at least under certain conditions.

Unlike rabbit eyes, in which retinal vasculature exists only in the medullary wing area, the $\mathrm{NB}$ obtained from the fundus in human eyes is affected by both retinal and choroidal circulation. ${ }^{20}$ In the case of Dutch rabbits, no significant difference was observed between the $\mathrm{NB}_{\text {av }}$ obtained using the diode laser from the medullary field where no discrete vessels were observed and the $\mathrm{NB}_{\mathrm{av}}$ from the extramedullary field, within an IOP range between 10 and $80 \mathrm{~mm} \mathrm{Hg}{ }^{12}$ This finding suggests that the contribution of retinal microcirculation to the NB obtained from the fundus using the diode laser is very minor in rabbits. Measurement of $\mathrm{NB}_{\mathrm{av}}$ obtained from the posterior fundus of monkeys using the diode laser before and after obstruction of a retinal artery (experimental branch retinal artery occlusion) suggested that the contribution of retinal microcirculation to $\mathrm{NB}_{\mathrm{av}}$ obtained with the diode laser was about $20 \%$ or less in monkeys. ${ }^{21}$ These results suggest that the contribution of choroidal circulation to the $\mathrm{NB}_{\mathrm{av}}$ obtained in the present study from the human fundus with no discrete retinal vessels visible is probably much greater than the contribution of retinal microcirculation. The NB obtained from the foveal area is supposed to be affected only by choroidal circulation because the area is free of retinal capillaries. It must be noted that the laser speckle method does not give an absolute value for the tissue blood velocity, but a relative value, because NB is also influenced by the reflectivity of the laser beam in the tissue. Therefore, the $\mathrm{NB}_{\text {av }}$ obtained from the $\mathrm{ONH}$, foveal area, or mid-peripheral fundus should not be directly compared and may not be suited for interindividual comparison. On the other hand, this method yields good reproducibility in the same tissue of an individual, ${ }^{18}$ and its change relative to the baseline correlates well with the relative change of blood flow determined using the microsphere method $^{12141522}$ or hydrogen gas clearance method. ${ }^{13}$ These characteristics and the 
simplicity of the measurements makes this method very useful in following the time course of change in an individual tissue.

EFFECT OF SCLERAL BUCKLING PROCEDURE

The present study indicated that segmental scleral buckling procedures with encircling elements significantly decreased $\mathrm{NB}_{\text {ch-ret }}$ on the buckled side. Using bidirectional laser Doppler in conjunction with retinal photography, Ogasawara $e t a l^{6}$ reported that in the patients who had undergone uncomplicated scleral buckling procedures the blood flow rates through the major temporal retinal arteries were on average $50 \%$ lower in the surgically treated eyes than in the unoperated contralateral eyes. If we assume that the contribution of retinal microcirculation to the $\mathrm{NB}_{\text {ch-ret }}$ obtained in the present study is approximately $20 \%$ according to the result reported by Isono, ${ }^{21}$ it seems difficult to explain a $40 \%$ decrease in the $\mathrm{NB}_{\text {ch-ret }}$ on the buckled side after scleral buckling only by its effect on retinal circulation. A decrease in choroidal tissue blood velocity after the scleral buckling procedure seems very likely. This finding is consistent with previous reports using the microsphere ${ }^{23}$ or hydrogen gas clearance method ${ }^{24}$ in rabbits that choroid and retina blood flow buckled with encircling elements was significantly decreased $^{23}$ or that choroidal blood flow was reduced at the posterior and peripheral sides of the encircling band. ${ }^{24}$ Ohkubo $^{25}$ found signs of reduced retinal perfusion in regions of reattached retina that were indented by scleral buckling procedures. Compressional mechanisms have been cited as the cause of reduced choroidal blood flow following scleral buckling procedures. ${ }^{92324}$ Thus, the reduced blood velocity in the choroid was probably attributed to direct obstruction of the choroidal venous drainage due to scleral buckling and/or local effects of cryotherapy.

The important finding in the present study is that the $\mathrm{NB}_{\text {ch-ret }}$ on the unbuckled side, the $\mathrm{NB}_{\text {fovea }}$, and $\mathrm{NB}_{\mathrm{ONH}}$ showed no significant change in spite of the significant decrease in $\mathrm{NB}_{\text {ch-ret }}$ on the buckled side. This result may not be consistent with those of previous reports suggesting substantial decrease in the posterior segment circulation on the whole at various intervals after buckling procedure ${ }^{5-11}$ - that is, $50 \%$ decrease in the flow rates in the major temporal retinal arteries, ${ }^{6} 35 \%-50 \%$ decrease in flow velocities in the central retinal artery, ${ }^{7}$ $31 \%$ decrease in the flow velocity in the ophthalmic artery, ${ }^{8}$ and $53 \%$ decrease in the ocular pulse. ${ }^{11}$ These previous reports, however, did not study the circulatory change in different parts of the fundus. Findings of the present study suggested that the segmental scleral buckling procedure with encircling elements does not cause clinically significant circulatory changes elsewhere on the buckled side, including the $\mathrm{ONH}$ and the foveal area where postoperative vision might be affected. The discrepancy between the present result and previous reports might be partly due to that the reduction of blood flow rate depends upon the degree of constriction of the encircling band, and/or the extent of the exoplant as reported by $\mathrm{Mano}^{24}$ or Diddie et al. ${ }^{23}$ In the present study, the encircling element was fastened with a moderate tightness and the extent of the exoplant was 1.5 or 2.5 quadrants. If the encircling element was fastened more tightly and/or the exoplant was more than three quadrants, it may be possible that not only the $\mathrm{NB}_{\text {ch-ret }}$ on the unbuckled side, but also $\mathrm{NB}_{\text {fovea }}$, and/or $\mathrm{NB}_{\mathrm{ONH}}$ are also affected significantly. In all of the present subjects, retinal detachment was successfully treated without any complications and it seems very unlikely that buckling and/or fastening of the encircling element were unreasonably mild. As discussed above, the present $\mathrm{NB}_{\text {ch-ret }}$ is thought to mainly reflect choroidal circulation, and partly retinal circulation. Therefore, the present result does not exclude the possibility that the retinal blood flow was slightly decreased also on the unbuckled side, as Ogasawara et $a l^{6}$ reported, while the $\mathrm{NB}_{\text {ch-ret }}$ showed no significant change. Whether the $\mathrm{NB}_{\text {fovea }}$ or $\mathrm{NB}_{\mathrm{ONH}}$ was also unaltered after segmental scleral buckling and encircling procedure in patients with pre-existing compromise of ocular tissue circulation awaits future study.

In summary, the present study demonstrated in otherwise healthy human eyes that the segmental scleral buckling procedure with encircling elements fastened with a moderate tightness causes a decrease in the blood velocity only in the choroid and/or retina on the buckled side, but little change in other areas of the fundus, including the $\mathrm{ONH}$, foveal area, and fundus on the unbuckled side.

This work was supported in part by grant in aid for developmental scientific research (B) No 01870074 from the Ministry of Education, Science, Sports, and Culture of Japan.

1 Boniuk M, Zimmerman LE. Necrosis of uvea, sclera, and retina following operations for retinal detachment. Arch Ophthalmol $1961 ; 66: 318-26$.

2 Foulds WS, Reid H, Chrisholm IA. Factors influencing visual recovery after retinal detachment surgery. Mod Probl visual recovery after retinal

3 Jarret WH, Brockhurst RJ. Unexplained blindness and optic atrophy following retinal detachment surgery. Arch Ophthalmol 1965;73:782-91

4 Robertson DM. Anterior segment ischemia after segmental episcleral buckling and cryopexy. Am $f$ Ophthalmol 1975;79:871-4

5 Yoshida A, Feke GT, Green GT, et al. Retinal circulatory changes after scleral buckling procedures. Am f Ophthalmol 1983;95:182-8

6 Ogasawara H, Feke GT, Yoshida A, et al. Retinal blood flow alterations associated with scleral buckling and encircling procedures. Br f Ophthalmol 1992;76:275-9.

7 Regillo CD, Sergott RC, Brown GC. Successful scleral buckling procedures decrease central retinal artery blood flow velocity. Ophthalmology 1993;100:1044-9.

8 Santos L, Capeans C, Gonzalez F, et al. Ocular blood flow Santos L, Capeans C, Gonzalez F, et al. Ocular blood flow
velocity reduction after buckling surgery. Graefes Arch Clin Exp Ophthalmol 1994;232:666-9.

9 Dobbie JG. Circulatory changes in the eye associated with retinal detachment and its repair. Trans Am Ophthalmol Soc 1980;78:503-66.

10 Johnson MW, Han DP, Hoffman KE. The effect of scleral buckling on ocular rigidity. Ophthalmology 1990;97:190-5.

11 Yoshida A, Hirokawa H, Ishiko S, et al. Ocular circulatory changes following scleral buckling procedures. $\mathrm{Br} \mathcal{F}$ Ophthalmol 1992;76:529-31.

12 Tamaki Y, Araie M, Kawamoto E, et al. Non-contact two-dimensional measurement of microcirculation in choroid and optic nerve head using laser speckle phenomenon. Exp Eye Res 1995;60:373-84.

13 Sugiyama T, Utsumi T, Azuma I, et al. Measurement of optic nerve head circulation: comparison of laser speckle and hydrogen clearance methods. Fpn f Ophthalmol 1996;40:339-43. 
14 Tamaki Y, Araie M, Kawamoto E, et al. Noncontact, two-dimensional measurement of retinal microcirculation using laser speckle phenomenon. Invest Ophthalmol Vis Sci 1994;35:3825-34.

15 Tamaki Y, Araie M, Tomita K, et al. Time change of nicardipine effet on choroidal circulation in rabbit eyes. Curr Eye Res 1996;15:543-8.

16 Fercher AF, Briers JD. Flow visualization by means of single-exposure speckle photography. Opt Commun 1981; 37:326-30

17 Briers JD, Fercher AF. Retinal blood-flow visualization by means of laser speckle photography. Invest Ophthalmol Vis Sci 1982;22:255-9.

18 Tamaki Y, Araie M, Tomita K, et al. Real-time measurements of tissue circulation in optic nerve head or choroid in human eyes using laser speckle phenomenon. $f p n$ f Ophthalmol 1997;4:49-54.
19 Koelle JS, Riva CE, Petrig BL, et al. Depth of tissue sampling in the optic nerve head using laser Doppler flowmetry. Lasers Med Sci 1993;8:49-54.

20 Isono H, Kimura Y, Aoyagi K. Analysis of choroidal blood flow by laser speckle flowgraphy. Nippon Ganka Gakkai Zasshi (F Jpn Ophthalmol Soc) 1997;101:684-91.

21 Isono H. Invest Ophthalmol Vis Sci 1997;38(Suppl):1050.

22 Tomidokoro A, Araie M, Tamaki Y, et al. In vivo measurement of iridial circulation using laser speckle phenomenon. Invest Ophthalmol Vis Sci 1998;39:364-71.

23 Diddie KR, Ernest JT. Uveal blood flow after $360^{\circ}$ constriction in the rabbit. Arch Ophthalmol 1980;78:503-66.

24 Mano T. Choroidal circulation after encircling procedure I. Immediate effect. Folia Ophthalmol 7pn 1982;33:460-7.

25 Ohkubo $\mathrm{H}$. Fluorescein angiographic findings in the detached and reattached retina. Fpn f Ophthalmol 1988;32:423-8. 